\title{
Quantum electrodynamics of resonance energy transfer in nanowire systems*
}

\author{
Dilusha Weeraddana, Malin Premaratne ${ }^{\dagger}$ \\ Advanced Computing and Simulation Laboratory $(A \chi L)$, \\ Department of Electrical and Computer Systems Engineering, \\ Monash University, Clayton, Victoria, 3800, Australia \\ David L. Andrews ${ }^{\ddagger}$ \\ School of Chemistry, University of East Anglia, Norwich Research Park, Norwich NR4 7TJ, United Kingdom
}

(Dated: February 5, 2016)

\begin{abstract}
Nonradiative resonance energy transfer (RET) provides the ability to transfer excitation energy between contiguous nanowires (NWs) with high efficiency under certain conditions. Nevertheless, the well established Förster formalism commonly used to represent RET was developed for energy transfer primarily between molecular blocks (i.e. from one molecule, or part of a molecule, to another). Although deviations from Förster theory for functional blocks such as NWs have been previously studied, the role of the relative distance, orientation of transition dipole moment pairs, and the passively interacting matter on electronic energy transfer, are to a large extent unknown. Thus, a comprehensive theory that models RET in NWs is required. In this context, analytical insights to give a deeper and more intuitive understanding of the distance and orientation dependence of RET in NWs is presented within the framework of quantum electrodynamics. Additionally, the influence of an included intermediary on the rate of excitation energy transfer is illustrated, embracing indirect energy transfer rate and quantum interference. The results deliver equations that afford new intuitions into the behavior of virtual photons. In particular, results indicate that RET efficiency in a NW system can be explicitly expedited or inhibited by a neighbouring mediator, depending on the relative spacing and orientation of NWs.
\end{abstract}

PACS numbers: $31.30 . \mathrm{J}-, 78.70 .-\mathrm{g}, 33.50 . \mathrm{Hv}, 78.67 . \mathrm{De}, 78.67 . \mathrm{Uh}, 78.67 . \mathrm{Hc}$

\section{INTRODUCTION}

Radiationless near-field transportation of energy from a donor particle, initially in its excited electronic state, to an acceptor in its ground state, is of considerable interest for diverse applications in science and engineering. Beyond wavefunction overlap, a compelling photo-physical process known as resonance energy transfer (RET) gains control. RET, also often known as electronic energy transfer (EET) [1], has been extensively exploited in artificial light harvesting antenna devices [2-4], spasers $[5,6]$ and especially in biology as a spectroscopic ruler to study conformational dynamics [7]. The study of RET in nanostructures has recently envisaged various prospective applications ranging from solar cell systems [8-10] to optical switching [11-14].

Nanotechnology offers means to study and fabricate nanostructures with large aspect ratios and small diameters, commonly termed nanowires (NWs) [15, 16]. These have been the focus of extensive research during the last few decades [17-20]. Their length is sufficiently large for easy manipulation as building blocks in fabricating superstructures. Electronic interactions between NWs at the nanoscale elevate the properties of a superstructure. Here, too, one of the important mechanisms for strong

\footnotetext{
$*$

† dilusha.weeraddana@monash.edu,malin.premaratne@monash.edu

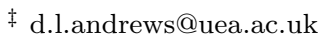

interaction is the RET, which results from Coulomb interaction between excitons confined in NWs [21-23].

Numerous studies have previously been reported on resonance energy transfer mechanism and related effects in systems consisted of NWs [24-26]. In many respects, the process of RET is well illustrated by semiclassical theories of radiationless energy transfer [26]. In this form of representation, the phenomenon is commonly considered as a first order perturbative process. However, in reality, RET processes are fully quantum mechanical in nature and they are formally described within the framework of quantum electrodynamics (QED) [27-33]. Here, the direct energy transfer emerges as a second-order process, mediated by the intermolecular propagation of virtual photons. The higher order reflects taking due account of causality and retardation; the electronic decay of one component and the resultant excitation of another, at a different point in space, cannot be simultaneous. Moreover, indirect energy transfer emerges via a vicinal neighbouring object occurs as a fourth-order process [34]. Therefore, QED has been widely applied to electronic coupling between the donor and acceptor over all distances, producing a unified theory that reconciles both RET and radiative energy transfer as the short- and long-range asymptotics of one mechanism [35, 36].

The main purpose of this paper is to investigate RET in NW systems analytically, by developing a comprehensive quantum electrodynamical analysis including statesequence methodology. Here, we extend our recent analysis [37], by relaxing uniaxial constraint imposed on the coupling photon. Of particular interest to this study is 
the distance and orientational dependence of a pair of NWs, embedded within another vicinal NW. Interestingly, a path is established towards a formalism that will allow the identification of specific attributes to expedite or inhibit electronic energy transfer, providing a detailed picture and understanding of RET in NWs. In Sec. II of this paper, the background molecular QED theory of RET is reviewed for both direct and third-body modified energy transfer. The direct and indirect RET for NWs are presented in Sec. III and Sec. IV respectively. Results are discussed in Sec.V followed by the conclusions in Sec. VI.

\section{EXCITATION ENERGY TRANSFER: QED PERSPECTIVE}

\section{A. System Hamiltonian}

It is appropriate to begin with the generic quantum energy operator for a system comprising of a number of particles and the radiation field, described by multipolar formulation of molecular QED. This Hamiltonian is expressible as follows [28]:

$$
H_{\text {total }}=\sum_{\xi} H_{\text {int }}(\xi)+\sum_{\xi} H_{\text {mat }}(\xi)+H_{\text {rad }}
$$

where $H_{\text {int }}(\xi)$ is the interaction Hamiltonian of the particle $\xi$ with the radiation field, $H_{m a t}(\xi)$ is the matter Hamiltonian of the particle $\xi$, and $H_{\text {rad }}$ represents the second-quantized radiation field Hamiltonian of the incident light field. The $H_{\text {int }}(\xi)$ describes interactions such as absorption and emission, (equally for either real or virtual photons), and it is described using the multipolar Hamiltonian in the dipole approximation [28]

$$
H_{i n t}=-\boldsymbol{\mu}(\xi) \cdot \boldsymbol{E}\left(\boldsymbol{R}_{\xi}\right)
$$

where the interaction Hamiltonian compromises contributions for each species $\xi$ located at $\boldsymbol{R}_{\xi}$, the $\boldsymbol{\mu}(\xi)$ is the electric-dipole moment operator and $\boldsymbol{E}\left(\boldsymbol{R}_{\xi}\right)$ is the operator for the electric displacement field at the specified location $\boldsymbol{R}_{\xi}$.

The transfer of energy beyond significant wavefunction overlap, generally entails a mechanism known as RET, mainly associated with electric dipole-electric dipole (E1-E1) coupling. In this event, an excited donor $(D)$ emits excitation energy which is transferred to an acceptor $(A)$; the donor falls back to its ground state while the acceptor is excited corresponds to the process

$D^{\alpha}+A^{0} \longrightarrow D^{0}+A^{\beta}$.

Here, superscripts denote donor and acceptor states. In the QED theory, this process is mediated by a virtual photon, coupling the donor decay and acceptor

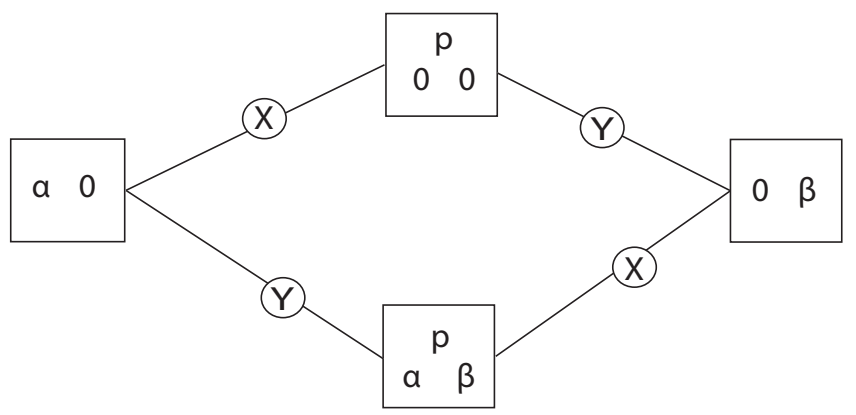

(a)

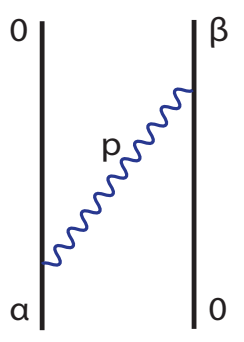

(b)

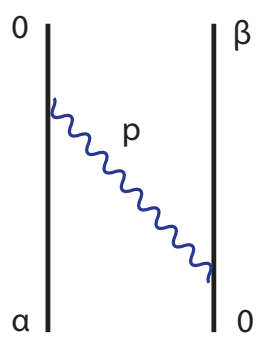

(c)
FIG. 1. State-sequence diagram (a) for direct RET. In each box, the state of donor $D$ is represented by the symbol on the left and the state of acceptor $A$ by the symbol on the right, $p$ denotes virtual photon. The Feynman diagrams show the time-order: (b) $(\mathrm{X}) \rightarrow(\mathrm{Y})$; (c) $(\mathrm{Y}) \rightarrow(\mathrm{X})$.

excitation through its creation and subsequent annihilation. The quantum amplitude for RET is based on a Schrödinger state vector representation of quantum dynamics, where matrix element for RET is represented as a sum of differently time-ordered contributions [28]. However, one can apply alternative formulations, for example, in terms of a density matrix in Liouville space [38]. The state sequence representation of the two time ordered contributions is depicted in Fig.1 (a) and the corresponding Feynman diagrams [39] are depicted in Fig.1 (b),(c). As shown in the figures, tracing the upper pathway; the virtual photon is created at $D$ and annihilated at $A$. The lower path depicts the case where virtual photon is created at $A$ and annihilated at $D$, as is also consistent with the time-energy uncertainty basis for conventional time orderings. As both paths lead to the same final state, calculation of the full RET quantum amplitude requires their summation.

\section{B. Direct energy transfer and the influence of an included intermediary}

The standard starting point for the development of the QED formalism of RET is the Fermi's golden rule rate equation. For a system proceeding from its initial state $I$ to final state $F$, the transfer rate (probability per unit 


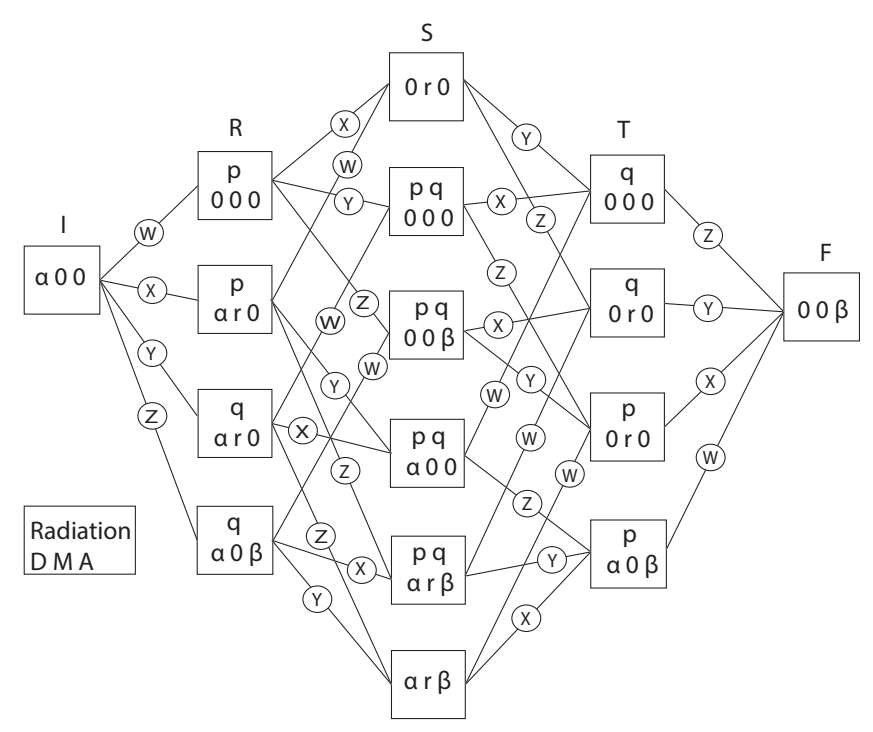

FIG. 2. State-sequence diagram for the indirect RET. Time progresses left to right, each of the 16 boxes representing one of the possible overall states of the system in one of the five stages $I, R, S, T, F, p, q$ denote virtual photons

time) is explicitly given by,

$$
\Gamma_{\text {tran }}=\frac{2 \pi}{\hbar}\left|M_{F I}\right|^{2} \rho
$$

where $\rho$ represents the density of final states, and $M_{F I}$ is the matrix element connecting the initial and final states of the system (sometimes loosely termed the quantum amplitude of the energy transfer process), which has the perturbation expansion

$$
\begin{array}{r}
M_{F I}=\left\langle F\left|H_{\text {int }}\right| I\right\rangle+\sum_{R} \frac{\left\langle F\left|H_{\text {int }}\right| R\right\rangle\left\langle R\left|H_{\text {int }}\right| I\right\rangle}{E_{I}-E_{R}}+ \\
\sum_{R, S} \frac{\left\langle F\left|H_{\text {int }}\right| R\right\rangle\left\langle R\left|H_{\text {int }}\right| S\right\rangle\left\langle S\left|H_{\text {int }}\right| I\right\rangle}{\left(E_{I}-E_{R}\right)\left(E_{I}-E_{S}\right)}+ \\
\sum_{R, S, T} \frac{\left\langle F\left|H_{\text {int }}\right| T\right\rangle\left\langle T\left|H_{\text {int }}\right| S\right\rangle\left\langle S\left|H_{\text {int }}\right| R\right\rangle\left\langle R\left|H_{\text {int }}\right| I\right\rangle}{\left(E_{I}-E_{R}\right)\left(E_{I}-E_{S}\right)\left(E_{I}-E_{T}\right)}+.
\end{array}
$$

where $I, F$ are initial, final states respectively and $R, S, T$ denote intermediate states. If $\zeta=I, F, R, S, T$, then $E_{\zeta}$ denotes the corresponding eigenenergy.

For the case of direct energy transfer, electronic energy transfer from a donor to an acceptor in the absence of a surrounding medium is calculated from the second term in the time-dependent perturbation series given in Eq. 4. Thus, the general formula for the direct interaction between two nano-particles can be expressed as

$$
\begin{array}{r}
M_{F I}^{d}=\frac{\mu_{n}^{0 \alpha}(D) \mu_{m}^{\beta 0}(A)}{2 V \epsilon_{0}} \sum_{a} \frac{E_{a n}^{*}\left(\boldsymbol{R}_{D}\right) E_{a m}\left(\boldsymbol{R}_{A}\right) p}{k-p}- \\
\frac{E_{a n}\left(\boldsymbol{R}_{D}\right) E_{a m}^{*}\left(\boldsymbol{R}_{A}\right) p}{k+p}
\end{array}
$$

Here a concise notation for the transition dipole moments is introduced, e.g. $\mu^{0 \alpha}(D) \equiv\left\langle D^{0}|\mu(D)| D^{\alpha}\right\rangle$, and $a=\{\boldsymbol{p}, \lambda\}$ represents the photonic modes, $V$ is an arbitrary quantization volume and $i, j$ are cartesian coordinates; also $p$ is the corresponding photon wave number which need not be equal to $k$.

The exchange of an additional virtual photon with an included intermediary is the lowest-order coupling process that promotes RET to third-body-mediated RET [40-42]. In our previous work, we have described how the four distinct matter-radiation interaction events (W, $\mathrm{X}, \mathrm{Y}, \mathrm{Z}$ in Fig. 2) modify the direct RET rate to thirdbody-modified RET [37], Fig. 2 illustrates the systems evolution through all five stages in one state-sequence diagram. Essentially, at each event, one particle undergoes a transition between states $0, \alpha, r, \beta$ and one photon is either created or annihilated by giving rise to $4 !=24$ Feynman diagrams. Therefore, the coupling matrix element for the case of third-body-mediated is calculated from the fourth term in the time dependent perturbation series stated in Eq. 4

$$
\begin{array}{r}
M_{F I}^{i}=-\sum_{a} \sum_{b}\left(\frac{\hbar c p}{2 V \epsilon_{0}}\right)\left(\frac{\hbar c q}{2 V \epsilon_{0}}\right) \mu_{n}^{0 \alpha}(D) \mu_{m}^{\beta 0}(A) \alpha_{k l}(M ; k) \\
\times\left\{\frac{E_{a n}\left(\boldsymbol{R}_{D}\right) E_{a k}^{*}\left(\boldsymbol{R}_{M}\right) E_{b m}\left(\boldsymbol{R}_{A}\right) E_{b l}^{*}\left(\boldsymbol{R}_{M}\right)}{(p-k)(q-k)}+\frac{E_{a n}\left(\boldsymbol{R}_{D}\right) E_{a k}^{*}\left(\boldsymbol{R}_{M}\right) E_{b m}^{*}\left(\boldsymbol{R}_{A}\right) E_{b l}\left(\boldsymbol{R}_{M}\right)}{(p-k)(q+k)}+\right. \\
\left.\frac{E_{a n}^{*}\left(\boldsymbol{R}_{D}\right) E_{a k}\left(\boldsymbol{R}_{M}\right) E_{b m}\left(\boldsymbol{R}_{A}\right) E_{b l}^{*}\left(\boldsymbol{R}_{M}\right)}{(p+k)(q-k)}+\frac{E_{a n}^{*}\left(\boldsymbol{R}_{D}\right) E_{a k}\left(\boldsymbol{R}_{M}\right) E_{b m}^{*}\left(\boldsymbol{R}_{A}\right) E_{b l}\left(\boldsymbol{R}_{M}\right)}{(p+k)(q+k)}\right\} \\
\alpha_{k l}(M ; k)=\sum_{r} \mu_{k}^{r 0}(M) \mu_{l}^{0 r}(M)\left\{\frac{1}{E_{r 0}-\hbar c k}+\frac{1}{E_{r 0}+\hbar c k}\right\}
\end{array}
$$




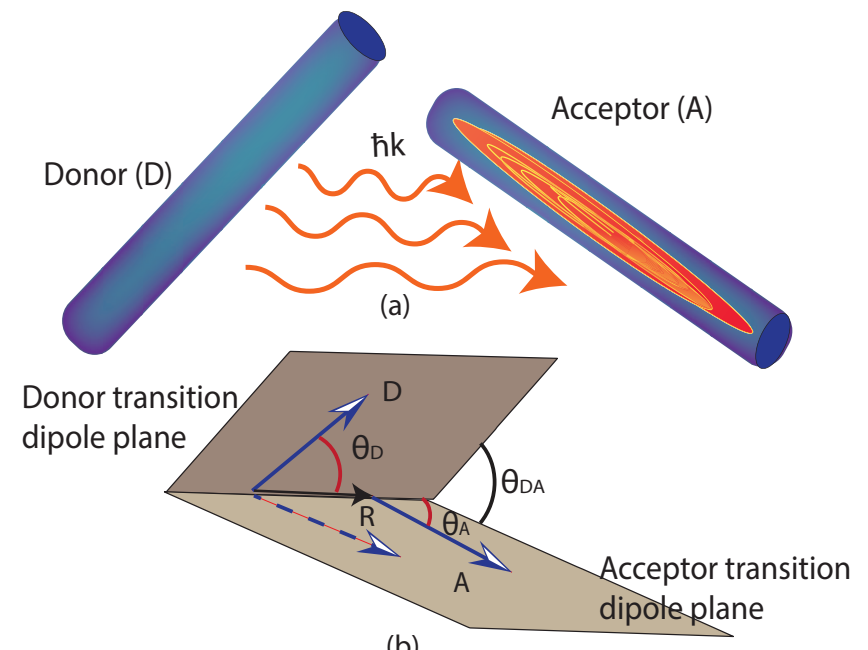

(b)

FIG. 3. Schematics for the direct resonance energy transfer (a) NW to NW; (b) the orientational factors in Eq.(17), $\theta_{D}$ and $\theta_{A}$ are the angles formed by the donor and acceptor transition dipole moments with respect to the displacement vector $\boldsymbol{R}$, and $\theta_{D A}$, the angle between the two transition dipole moments.

Here, also, $\alpha_{k l}(M ; k)$ is the dynamic polarizability of particle $M[43,44]$.

The total matrix element is given by the sum of second and fourth terms in Eq. (4), and the transfer rate is then seen to be a sum of three terms, namely

$$
\begin{aligned}
\Gamma_{\text {tran }}^{\text {total }} & =\frac{2 \pi}{\hbar}\left|M_{F I}^{d}+M_{F I}^{i}\right|^{2} \rho \\
& =\frac{2 \pi}{\hbar}\left[\left|M_{F I}^{d}\right|^{2}+\left|M_{F I}^{i}\right|^{2}+2 \operatorname{Re} \bar{M}_{F I}^{d} M_{F I}^{i}\right] \rho
\end{aligned}
$$

where the third term is a quantum interference contribution to the rate arising from both direct and indirect mechanisms.

\section{DIRECT COUPLING OF TWO NANOWIRE SYSTEM}

We consider a system which comprises of the radiation field, and two NWs of length $L$ separated by a distance $R$ (center to center separation) as depicted in Fig. 3 (a). In contrast to our previous work [37], the significance is on removal of the directional constraint on the coupling photon. Owing to the cylindrical symmetry of NWs, it is convenient to model EM waves using Hankel function of or$\operatorname{der} n[25,45,46]: \boldsymbol{e}^{(\lambda)}(\boldsymbol{p}) \sum_{n} H_{n}(p R) e^{i n \alpha}$, where $\boldsymbol{e}^{(\lambda)}(\boldsymbol{p})$ is the polarization vector $R$ and $\alpha$ are the radial and angular coordinates respectively. Directly substituting into Eq. (5) and converting the discrete summation over virtual photon wave vector $[25,46], \sum_{p} \Rightarrow \int \frac{A}{(2 \pi)^{2}} d^{2} \boldsymbol{p}$ to an integral yields

$$
\begin{aligned}
M_{F I}^{d}= & \frac{\mu_{n}^{0 \alpha}(D) \mu_{m}^{\beta 0}(A)}{8 \pi^{2} L \epsilon_{0}}\left(-\nabla^{2} \delta_{n m}+\nabla_{n} \nabla_{m}\right) \\
& \int_{0}^{\infty} \int_{0}^{2 \pi} \frac{H_{0}^{(1)}(p R)}{k-p}-\frac{H_{0}^{(2)}(p R)}{k+p} d \phi d p
\end{aligned}
$$

Expanding the Hankel function, performing contour integration and by the residue theorem

$$
\begin{array}{r}
M_{F I}^{d}=\frac{\mu_{n}^{0 \alpha}(D) \mu_{m}^{\beta 0}(A)}{2 \pi L R^{2} \epsilon_{0}}\left(-\nabla^{2} \delta_{n m}+\nabla_{n} \nabla_{m}\right) \\
\oint_{c} \frac{i k R Y_{0}(c)+c^{2} J_{0}(k R)}{(k R-c)(k R+c)} d c \\
M_{F I}^{d}=\frac{\mu_{n}^{0 \alpha}(D) \mu_{m}^{\beta 0}(A)}{4 L \epsilon_{0}}\left(-\nabla^{2} \delta_{n m}+\nabla_{n} \nabla_{m}\right) \\
\left\{Y_{0}(k R)-i J_{0}(k R)\right\}
\end{array}
$$

where $Y_{0}(k R), J_{0}(k R)$ are zero order second and first kind of Bessel functions respectively.

$$
\begin{array}{r}
M_{F I}^{d}=\frac{\mu_{n}^{0 \alpha}(D) \mu_{m}^{\beta 0}(A)}{4 L \epsilon_{0}}\left[k \delta_{n m}\left\{-Y_{2}(k R)+\frac{Y_{1}(k R)}{k R}\right\}-k\left\{Y_{1}(k R)\left(\frac{\delta_{n m}-\hat{R}_{n} \hat{R}_{m}}{R}\right)+\right.\right. \\
\left.k \hat{R}_{n} \hat{R}_{m}\left(-Y_{2}(k R)+\frac{Y_{1}(k R)}{k R}\right)\right\}-i k \delta_{n m}\left\{-J_{2}(k R)+\frac{J_{1}(k R)}{k R}\right\}+i k\left\{J_{1}(k R)\left(\frac{\delta_{n m}-\hat{R}_{n} \hat{R}_{m}}{R}\right)+\right. \\
\left.\left.k \hat{R}_{n} \hat{R}_{m}\left(-J_{2}(k R)+\frac{J_{1}(k R)}{k R}\right)\right\}\right]
\end{array}
$$

We now deploy the asymptotic series for $0<k R \ll \sqrt{ } n+$ 1 and $n \neq 0[47]$

$$
Y_{n}(k R) \sim-\frac{\Gamma(n)}{\pi}\left(\frac{2}{k R}\right)^{n}+\frac{1}{\Gamma(n+1)}\left(\frac{k R}{2}\right)^{n} \cot (n \pi)
$$




$$
J_{n}(k R) \sim \frac{1}{\Gamma(n+1)}\left(\frac{k R}{2}\right)^{n}
$$

By applying near-field limits $0<k R \ll 1$ on Eq.12 yields

$$
\begin{aligned}
M_{F I}^{d}= & \frac{\mu_{n}^{0 \alpha}(D) \mu_{m}^{\beta 0}(A)}{4 L \epsilon_{0}}\left\{\frac{\Gamma(1)}{\pi}\left(\frac{2}{k R}\right)\left(\frac{\delta_{n m}-\hat{R}_{n} \hat{R}_{m}}{R}\right)\right. \\
& \left.+k \hat{R}_{n} \hat{R}_{m}\left(-\frac{\Gamma(2)}{\pi}\left(\frac{2}{k R}\right)^{2}+\frac{\Gamma(1)}{\pi k R}\left(\frac{2}{k R}\right)\right)\right\}
\end{aligned}
$$

where $\Gamma(n)$ is the standard Gamma function which is expressed in $\int_{0}^{\infty} t^{n-1} e^{-t} d t=(n-1) \Gamma(n-1)$. Therefore, Eq.15 becomes

$$
\begin{aligned}
M_{F I}^{d} & =\frac{\mu_{n}^{0 \alpha}(D) \mu_{m}^{\beta 0}(A)}{2 \pi L R^{2} \epsilon_{0}}\left(\delta_{n m}-2 \hat{R}_{n} \hat{R}_{m}\right) \\
& =\frac{\kappa_{D A}\left|\mu_{n}^{0 \alpha}(D)\right|\left|\mu_{m}^{\beta 0}(A)\right|}{2 \pi L R^{2} \epsilon_{0}}
\end{aligned}
$$

where $\left|\kappa_{D A}\right|^{2}$ is an orientation factor. $\left|\kappa_{D A}\right|^{2}$ describes the influence of the relative orientations of the transition dipole moments of the donor and acceptor NWs, as given by

$$
\begin{aligned}
\kappa_{D A} & =\hat{\mu}_{n}^{0 \alpha}(D)\left(\delta_{n m}-2 \hat{R}_{n} \hat{R}_{m}\right) \hat{\mu}_{m}^{\beta 0}(A) \\
& =\cos \left(\theta_{D A}\right)-2 \cos \left(\theta_{D}\right) \cos \left(\theta_{A}\right)
\end{aligned}
$$

where $\theta_{D}$ is the angle between donor and separation vector $(\boldsymbol{R})$, and $\theta_{A}$ is the angle between acceptor and $\boldsymbol{R}$. $\theta_{D A}$ is the angle between donor and acceptor NWs (Fig. $3(\mathrm{~b}))$.

The energy transfer rate can be obtained from Eq. (3) and emerges in the following simple form:

$$
\Gamma_{\text {tran }}^{d}=\frac{\left|\kappa_{D A}\right|^{2}\left|\mu_{n}^{0 \alpha}(D)\right|^{2}\left|\mu_{m}^{\beta 0}(A)\right|^{2} \rho}{2 \pi L^{2} R^{4} \epsilon_{0}^{2} \hbar}
$$

\section{A. Distance dependance}

From Eq. (18), it is observed that the direct energy transfer in NWs exhibits an inverse fourth power dependence on the separation distance. This is because quantum amplitude is inversely proportional to the spacing between donor and acceptor $\left(M_{F I}^{d} \propto R^{-2}\right)$, and the energy transfer rate is proportional to the square modulus of the quantum amplitude of RET process $\left(\Gamma_{\text {tran }} \propto\left|M_{F I}^{d}\right|^{2}\right)$. The plots of Eq. (16), Eq. (18) are shown in Fig. 4 and Fig. 5 respectively. In the development of the plots, the following values were used [48]: $\left|\mu^{0 \alpha}(D)\right|=\left|\mu^{\beta 0}(A)\right|=5 \times 10^{-30} \mathrm{C} \mathrm{m} ; \rho=2 \times 10^{25}$ $\mathrm{J}^{-1}$. Fig. 4 (a) shows the functional dependence of resonant dipole-dipole interaction (RDDI) for various values of the donor-acceptor separation distance $(R)$. It is observed that due to the behaviour of the virtual photon propagation in a $2 \mathrm{D}$ realm, the RDDI decreases with $R$ by following an inverse square power dependence, resulting in a gradual decline of the energy transfer efficiency with the distance as illustrated in Fig. 5 (a). Fig. 4 (b) and Fig. 5 (b) compares the NW-to-NW coupling and transfer efficiency with quantum dot (QD) to $\mathrm{QD}$ as a function of $R$, where distance dependance of latter is sharper than former, signifying the high physical loses in the transmission medium due to the spherical symmetry of the QD.

\section{B. Orientational dependence}

The relative orientation of the donor and acceptor, and their individual orientations with respect to relative separation vector (see Fig. $3(\mathrm{~b})$ ), influence the RDDI in Eq.(16) in a variety of different ways, as depicted in Fig. 4 (c). Here, we explore the variation of RDDI at different $\theta_{D}$ values ranging from 0 to $\frac{\pi}{2}$ for fixed $\theta_{D A}$ (at 0 and $\pi), \theta_{A}$ (at 0 ). The coupling matrix element reaches its peak when the donor the is orthogonal to the displacement vector $\left(\theta_{D}=\frac{\pi}{2}\right), \theta_{D A}=0$ and also when $\theta_{D}=0$, $\theta_{D A}=\pi$. Further, it becomes minimum when $\theta_{D}=\frac{\pi}{2}$, $\theta_{D A}=\pi$ and also $\theta_{D}=0, \theta_{D A}=0$. Therefore, the transfer rate strongly depends on the orientation factor. It is clear from the Eq.(17) that $\kappa^{2}$ does not does not change if following operations are performed [29]:

1. flip the donor transition moment $\left(\hat{\mu}^{0 \alpha}(D) \rightarrow\right.$ $\left.-\hat{\mu}^{0 \alpha}(D)\right)$,

2. flip the acceptor transition moment $\left(\hat{\mu}^{\beta 0}(A) \rightarrow\right.$ $\left.-\hat{\mu}^{\beta 0}(A)\right)$,

3. allow the donor and acceptor to trade places $(R \rightarrow$ $-R)$

4. interchange the donor and acceptor transition moments $\left(\hat{\mu}^{0 \alpha}(D) \leftrightarrow \hat{\mu}^{\beta 0}(A)\right)$.

The angles $\theta_{D A}, \theta_{D}, \theta_{A}$ are dependent on one another. Therefore, we consider three cases to study the orientational dependence of RET rate.

1. case 1: $\theta_{D A}=0, \theta_{D}=0, \theta_{A}=0 ; \kappa_{D A}=-1$, (Fig. $6(\mathrm{a}))$

2. case 2: $\theta_{D A}=0, \theta_{D}=\frac{\pi}{2}, \theta_{A}=\frac{\pi}{2} ; \kappa_{D A}=1$, (Fig. $6(\mathrm{~b}))$

3. case 3: $\theta_{D A}=\frac{\pi}{2}, \theta_{D}=\frac{\pi}{2}, \theta_{A}=\frac{\pi}{2} ; \kappa_{D A}=0$, (Fig. $6(\mathrm{c}))$

As can be anticipated, the orientation factor becomes most favourable when the transition dipole moments are 


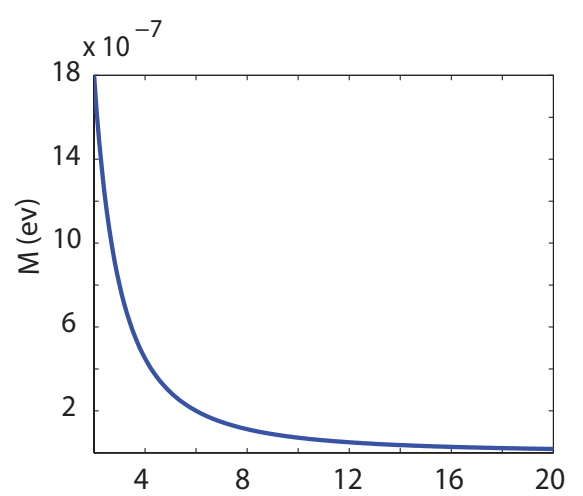

$\mathrm{R}(\mathrm{nm})$

(a)

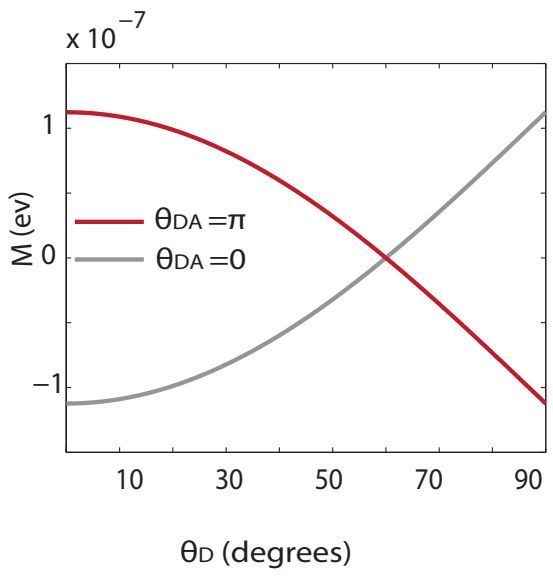

(c)

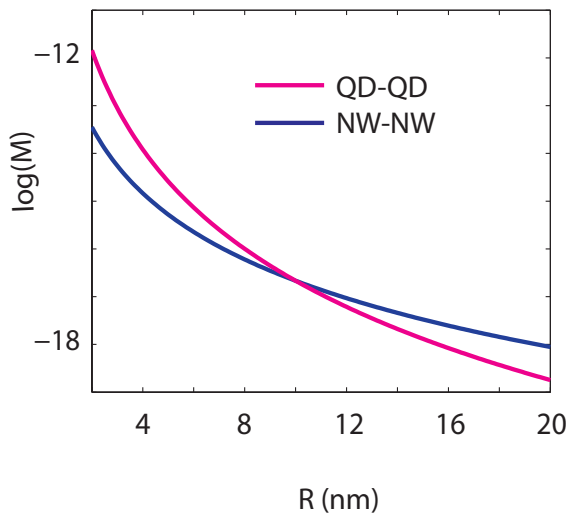

(b)
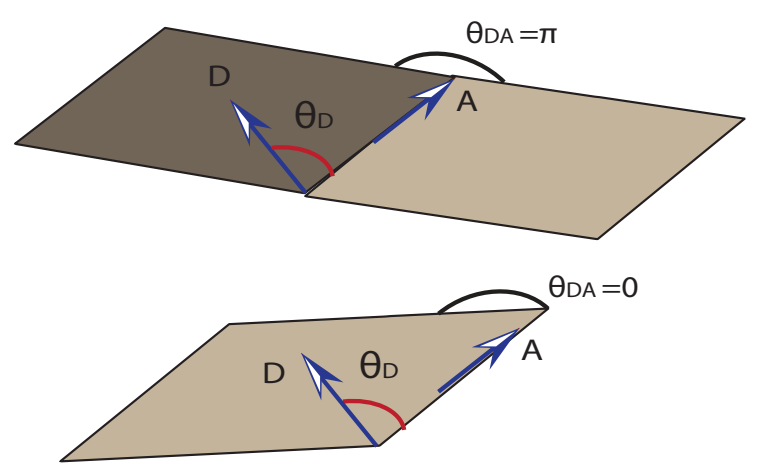

(d)

FIG. 4. RDDI strength in direct RET: (a) as a function of the relative distance $(R$ ) between $D$ and $A$; (b) NW to NW and QD to QD with respect to $R$; (c) variation as a function of $\theta_{D}$ when $\theta_{D A}=0$ and $\theta_{D A}=\pi$; (d) corresponding schematics for the relative orientations depicted in (c).

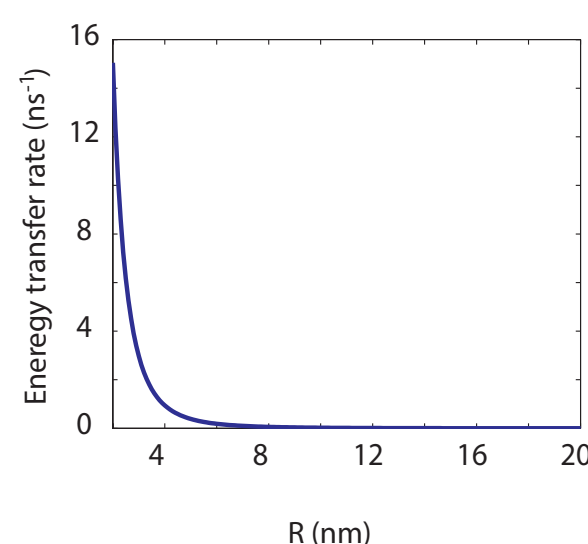

(a)

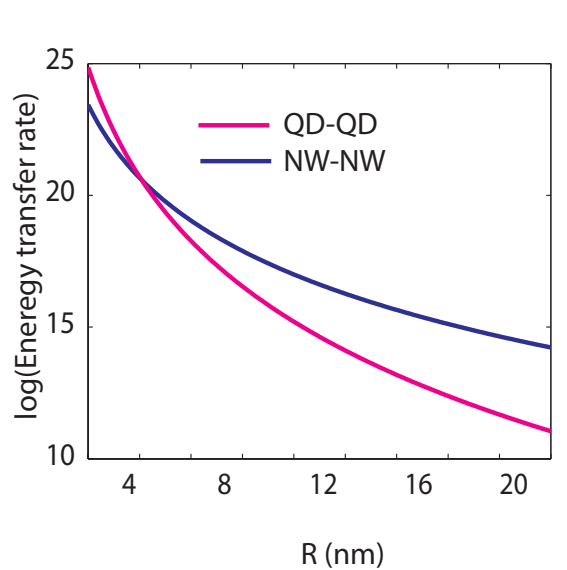

(b)

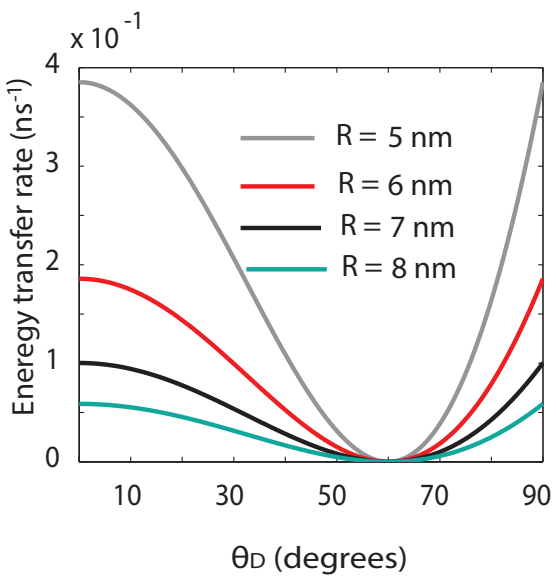

(c)

FIG. 5. Direct RET rate: (a) as a function of the relative distance $(R$ ) between $D$ and $A$; (b) NW to NW and QD to QD with respect to $R$; (c) as a function of $\theta_{D}$ for four different relative distances $(5 \mathrm{~nm}, 6 \mathrm{~nm}, 7 \mathrm{~nm}, 8 \mathrm{~nm}$ ) between D and A. 


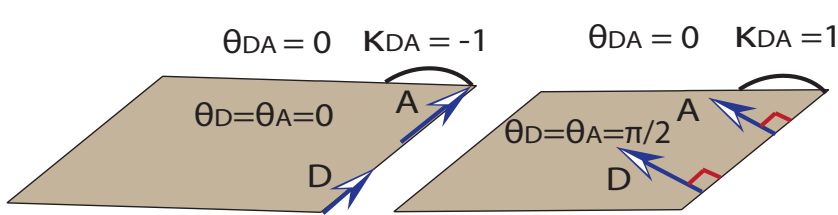

(a)

(b)

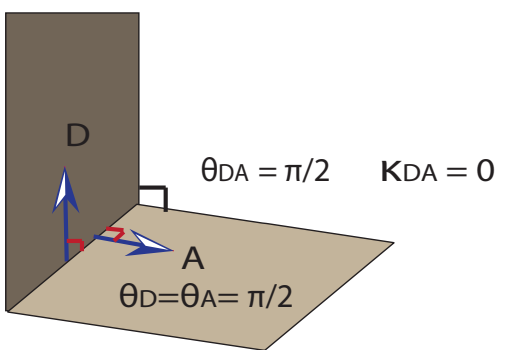

(c)

FIG. 6. Direct RET orientation factor in Eq.(17): (a) $\theta_{D A}=$ $0, \theta_{D}=0, \theta_{A}=0, \kappa_{D A}=-1$; (b) $\theta_{D A}=0, \theta_{D}=\frac{\pi}{2}, \theta_{A}=\frac{\pi}{2}$, $\kappa_{D A}=1$; (c) $\theta_{D A}=\frac{\pi}{2}, \theta_{D}=\frac{\pi}{2}, \theta_{A}=\frac{\pi}{2}, \kappa_{D A}=0$ parallel (or anti-parallel) to one another and to the displacement vector. This is classic Förster behaviour. Additionally, the coupling may also be prohibited between NWs by arranging them such that the transition dipole moments are mutually orthogonal. We further investigate this factor by keeping $\theta_{D A}, \theta_{A}$ fixed at 0 and varying $\theta_{D}$ from 0 to $\frac{\pi}{2}$. Interestingly, the orientation dependence of rate becomes weaker as the separation distance between donor and acceptor decreases, which is illustrated in Fig.5 (c). This is similar to the numerous studies reported for molecules and QDs discussed in [49, 50], with one important difference. Due to the cylindrical symmetry and the physical nature of the exchanged photon virtue in the $2 \mathrm{D}$ geometry, the orientation factor varies from $0 \leq \kappa^{2} \leq 1$

\section{NANOWIRE TO NANOWIRE RET IN THE VICINITY OF ANOTHER NANOWIRE}

We now insert a third NW, $M$, which acts as a bridge species between $D$ and $A$ as shown in Fig.7 (a). $M$ is engaged in relaying energy between donor and acceptor, but otherwise remains unchanged overall. Direct substitution into Eq. (6) yields

$$
\begin{array}{r}
M_{F I}^{i}=-\frac{\mu_{n}^{0 \alpha}(D) \mu_{m}^{\beta 0}(A)}{\left(2 V \epsilon_{0}\right)^{2}} \sum_{\boldsymbol{p}} \sum_{\boldsymbol{q}} \boldsymbol{e}_{n}^{(\lambda)}(\boldsymbol{p}) \overline{\boldsymbol{e}}_{l}^{(\lambda)}(\boldsymbol{p}) \boldsymbol{e}_{m}^{(\lambda)}(\boldsymbol{q}) \overline{\boldsymbol{e}}_{k}^{(\lambda)}(\boldsymbol{q}) \alpha_{k l}(M ; k) p^{2} q^{2} \times \\
\left\{\frac{H_{0}^{(2)}\left(p R^{\prime}\right) H_{0}^{(1)}\left(q R^{\prime \prime}\right)}{(p-k)(q-k)}+\frac{H_{0}^{(2)}\left(p R^{\prime}\right) H_{0}^{(2)}\left(q R^{\prime \prime}\right)}{(p-k)(q+k)}+\frac{H_{0}^{(1)}\left(p R^{\prime}\right) H_{0}^{(1)}\left(q R^{\prime \prime}\right)}{(p+k)(q-k)}+\frac{H_{0}^{(1)}\left(p R^{\prime}\right) H_{0}^{(2)}\left(q R^{\prime \prime}\right)}{(p+k)(q+k)}\right\}
\end{array}
$$

where $\boldsymbol{R}^{\prime}=\boldsymbol{R}_{M}-\boldsymbol{R}_{D}$ and $\boldsymbol{R}^{\prime \prime}=\boldsymbol{R}_{A}-\boldsymbol{R}_{M}$, so that $\boldsymbol{R}=\boldsymbol{R}_{A}-\boldsymbol{R}_{D}$.

$$
\begin{array}{r}
M_{F I}^{i}=-\frac{\mu_{n}^{0 \alpha}(D) \mu_{m}^{\beta 0}(A)}{\left(2 V \epsilon_{0}\right)^{2}} \sum_{\boldsymbol{p}} \sum_{\boldsymbol{q}} \boldsymbol{e}_{n}^{(\lambda)}(\boldsymbol{p}) \overline{\boldsymbol{e}}_{l}^{(\lambda)}(\boldsymbol{p}) \boldsymbol{e}_{m}^{(\lambda)}(\boldsymbol{q}) \overline{\boldsymbol{e}}_{k}^{(\lambda)}(\boldsymbol{q}) \alpha_{k l}(M ; k) p^{2} q^{2} \times \\
\left(\frac{H_{0}^{(2)}\left(p R^{\prime}\right)}{p-k}+\frac{H_{0}^{(1)}\left(p R^{\prime}\right)}{p+k}\right) \times\left(\frac{H_{0}^{(1)}\left(q R^{\prime \prime}\right)}{q-k}+\frac{H_{0}^{(2)}\left(q R^{\prime \prime}\right)}{q+k}\right)
\end{array}
$$

By converting the discrete summation over $p, q$ to an integral yields

$$
\begin{array}{r}
M_{F I}^{i}=-\frac{\mu_{n}^{0 \alpha}(D) \mu_{m}^{\beta 0}(A)}{\left(8 \pi^{2} L \epsilon_{0}\right)^{2}}\left(-\nabla^{2} \delta_{n l}+\nabla_{n} \nabla_{l}\right)\left(-\nabla^{2} \delta_{m k}+\nabla_{m} \nabla_{k}\right) \int_{0}^{\infty} \int_{0}^{2 \pi} \int_{0}^{\infty} \int_{0}^{2 \pi} \alpha_{k l}(M ; k) \times \\
\left(\frac{H_{0}^{(2)}\left(p R^{\prime}\right)}{p-k}+\frac{H_{0}^{(1)}\left(p R^{\prime}\right)}{p+k}\right) \times\left(\frac{H_{0}^{(1)}\left(q R^{\prime \prime}\right)}{q-k}+\frac{H_{0}^{(2)}\left(q R^{\prime \prime}\right)}{q+k}\right) d q d \phi d p d \varphi
\end{array}
$$

In a similar manner as the previous case in Sec. (III) the quantum amplitude, on performing contour integra- tion and using the residue theorem twice over two virtual photons, becomes 


$$
\begin{array}{r}
M_{F I}^{i}=-\frac{\mu^{0 \alpha}(D) \mu^{\beta 0}(A) \alpha_{k l}(M ; k)}{\left(4 L \epsilon_{0}\right)^{2}}\left[k \delta_{n l}\left\{-Y_{2}\left(k R^{\prime}\right)+\frac{Y_{1}\left(k R^{\prime}\right)}{k R}\right\}-k\left\{Y_{1}\left(k R^{\prime}\right)\left(\frac{\delta_{n l}-\hat{R_{n}^{\prime}}}{R^{\prime}} \hat{R}_{l}^{\prime}\right)+k \hat{R_{n}^{\prime}} \hat{R}_{l}^{\prime}\left(-Y_{2}\left(k R^{\prime}\right)\right.\right.\right. \\
\left.\left.\left.+\frac{Y_{1}\left(k R^{\prime}\right)}{k R^{\prime}}\right)\right\}-i k \delta_{n l}\left\{-J_{2}\left(k R^{\prime}\right)+\frac{J_{1}\left(k R^{\prime}\right)}{k R^{\prime}}\right\}+i k\left\{J_{1}\left(k R^{\prime}\right)\left(\frac{\delta_{n l}-\hat{R_{n}^{\prime}}}{R^{\prime}}\right)+k \hat{R_{n}^{\prime}} \hat{R}_{l}^{\prime}\left(-J_{2}\left(k R^{\prime}\right)+\frac{J_{1}\left(k R^{\prime}\right)}{k R^{\prime}}\right)\right\}\right] \\
{\left[k \delta_{m k}\left\{-Y_{2}\left(k R^{\prime \prime}\right)+\frac{Y_{1}\left(k R^{\prime \prime}\right)}{k R^{\prime \prime}}\right\}-k\left\{Y_{1}\left(k R^{\prime \prime}\right)\left(\frac{\delta_{m k}-\hat{R_{m}^{\prime \prime}}}{R} \hat{R_{k}^{\prime \prime}}\right)+k \hat{R_{m}^{\prime \prime}} \hat{R_{k}^{\prime \prime}}\left(-Y_{2}\left(k R^{\prime \prime}\right)\right.\right.\right.} \\
\left.\left.\left.+\frac{Y_{1}\left(k R^{\prime \prime}\right)}{k R^{\prime \prime}}\right)\right\}-i k \delta_{m k}\left\{-J_{2}\left(k R^{\prime \prime}\right)+\frac{J_{1}\left(k R^{\prime \prime}\right)}{k R^{\prime \prime}}\right\}+i k\left\{J_{1}\left(k R^{\prime \prime}\right)\left(\frac{\delta_{m k}-\hat{R_{j}^{\prime \prime}} \hat{R}_{k}^{\prime \prime}}{R^{\prime \prime}}\right)+k \hat{R_{m}^{\prime \prime}} \hat{R_{k}^{\prime \prime}}\left(-J_{2}\left(k R^{\prime \prime}\right)+\frac{J_{1}\left(k R^{\prime \prime}\right)}{k R^{\prime \prime}}\right)\right\}\right]
\end{array}
$$

The Eq.(22) for matrix element can be simplified by imposing near-field limits and using Eq.(13), (14)

$$
\begin{array}{r}
M_{F I}^{i}=-\frac{\mu^{0 \alpha}(D) \mu^{\beta 0}(A)}{\left(2 \pi L R^{\prime} R^{\prime \prime} \epsilon_{0}\right)^{2}}\left[\left(\delta_{n l}-2 \hat{R_{n}^{\prime}} \hat{R}_{l}^{\prime}\right)\left(\delta_{m k}-2 \hat{R_{m}^{\prime \prime}} \hat{R}_{k}^{\prime \prime}\right)\right. \\
\left.\alpha_{k l}(M ; k)\right]
\end{array}
$$

Therefore, in the presence of a neighbouring mediator, the matrix element for the mechanism of RET is duly modified to Eq.(23), which can also be written in the following form

$$
M_{F I}^{i}=-\frac{\left|\mu^{0 \alpha}(D)\right|\left|\mu^{\beta 0}(A)\right| \kappa_{D M} \kappa_{M A}}{\left(2 \pi L R^{\prime} R^{\prime \prime} \epsilon_{0}\right)^{2}}\left|\alpha_{k l}(M ; k)\right|
$$

where $\kappa_{D M}, \kappa_{M A}$ are expressed as

$$
\begin{aligned}
\kappa_{D M} & =\hat{\mu}^{0 \alpha}(D)\left(\delta_{n l}-2 \hat{R_{n}^{\prime}} \hat{R}_{l}^{\prime}\right) \hat{\mu}^{r 0}(M) \\
& =\cos \left(\theta_{D M}\right)-2 \cos \left(\theta_{D}^{\prime}\right) \cos \left(\theta_{M}\right) \\
\kappa_{M A} & =\hat{\mu}^{0 r}(M)\left(\delta_{m k}-2 \hat{R_{m}^{\prime \prime}} \hat{R}_{k}^{\prime \prime}\right) \hat{\mu}^{\beta 0}(A) \\
& =\cos \left(\theta_{M A}\right)-2 \cos \left(\theta_{M}^{\prime}\right) \cos \left(\theta_{A}^{\prime}\right)
\end{aligned}
$$

in which $\theta_{D}^{\prime}$ is the angle between $\mu(D)$ and $D$ - $M$ separation vector $\left(\boldsymbol{R}^{\prime}\right)$, and $\theta_{A}^{\prime}$ is the angle between $\mu(A)$ and $\boldsymbol{R}^{\prime \prime}$. $\theta_{D M}$ is the angle between $\mu(D)$ and $\mu(M), \theta_{M A}$ is the angle between $\mu(M)$ and $\mu(A) . \theta_{M}, \theta_{M}^{\prime}$ are angles formed by $\mu(M)$ with respect to $\boldsymbol{R}^{\prime}$ and $\boldsymbol{R}^{\prime \prime}$ (Fig. 7 (b)).

The application of the Fermi's golden rule now gives rise to the following expression for the transfer rate

$$
\Gamma_{\text {tran }}^{i}=\frac{\left.\left.\left|\mu^{0 \alpha}(D)\right|^{2}\left|\mu^{\beta 0}(A)\right|^{2}|| \kappa_{D M}\right|^{2}\left|\kappa_{M A}\right|^{2} \alpha_{k l}(M ; k)\right|^{2} \rho}{8 \pi \hbar\left(L^{2} R^{\prime 2} R^{\prime \prime 2} \epsilon_{0}^{2}\right)^{2}}
$$

Ideally, the result in Eq. (27) should be used to evaluate the indirect contribution to the RET rate mediated by neighbouring body $M$ in near field regime. Undoubtedly, the key factors are the position, orientation, and the trace polarizability of the passive NW.

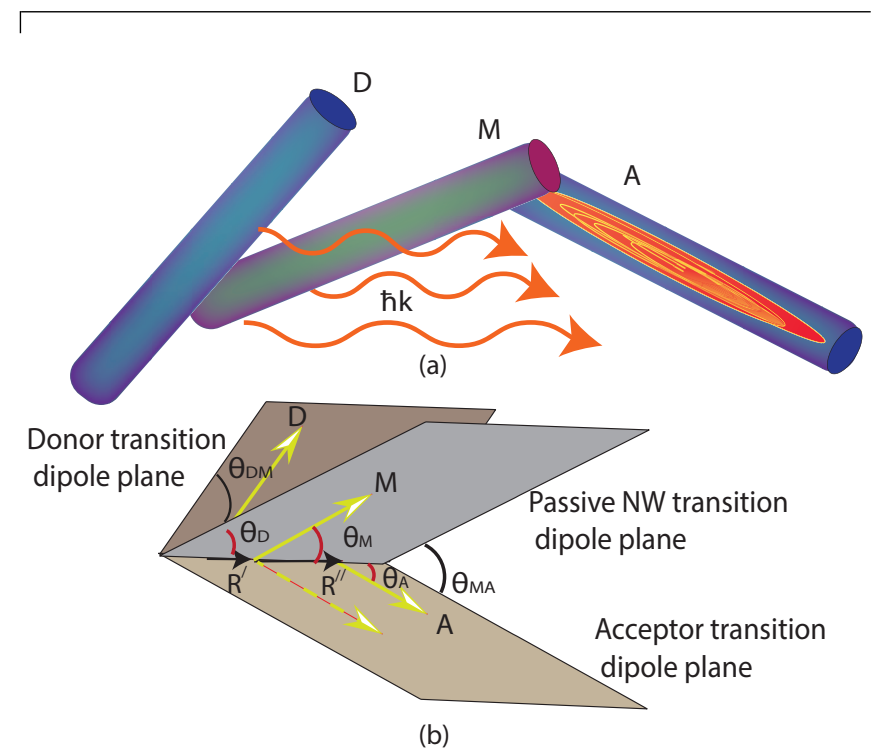

FIG. 7. Schematics for the third-body modified resonance energy transfer of (a) NW to NW; (b) the orientational factors in Eq.(25), (26), $\theta_{D}$, the angle between the donor and the displacement vector, $\theta_{A}$, the angle between the acceptor and the displacement vector. $\theta_{D M}$, is the angle between the $D, M$ transition dipole moments and $\theta_{M A}$, the angle between the $M, A$ transition dipole moments

In the development of the plots in Sec. (IV), the following values were used [48]: $\left|\mu^{0 \alpha}(D)\right|=\left|\mu^{\beta 0}(A)\right|=$ $5 \times 10^{-30} \mathrm{C} \mathrm{m} ; \rho=2 \times 10^{25} \mathrm{~J}^{-1}$. Again, $\alpha_{k l}(M ; k)$ is the ground state dynamic polarizability of the passive nanostructure. Polarizability values are related to refractive index by the Clausius- Mossotti equation, and an in-depth analysis of relevance to the current application has been carried out in [41, 43, 44]. Therefore, in the development of the graphs, we assume $\left|\alpha_{k l}(M ; k)\right|$ of the included intermediary takes a typical value of $25 \times 10^{-35} \mathrm{~J}^{-1} \mathrm{C}^{2} \mathrm{~m}^{2}$ $[42]$.

It is important to note that, values of $R, R^{\prime}, R^{\prime \prime}<1$ $\mathrm{nm}$ will generally signify the possibility of wave function overlap. In this regime, an alternative mode of energy transfer occurs, called the Dexter mechanism, and it is predicted to have an essentially exponential dependence on separation distance. In the present study, we restrict ourselves to separations where RET processes are me- 


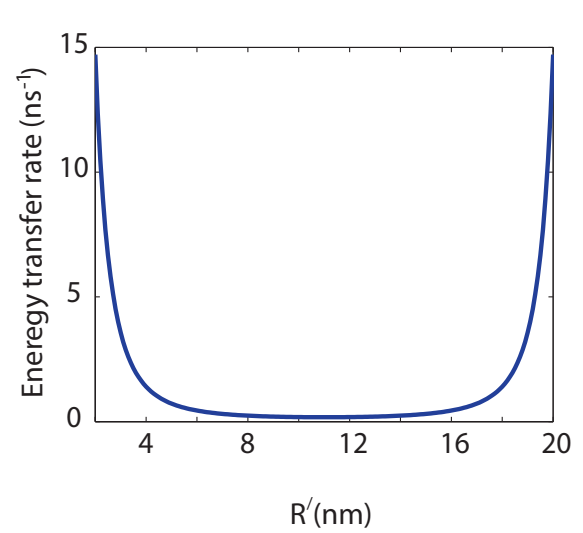

(a)

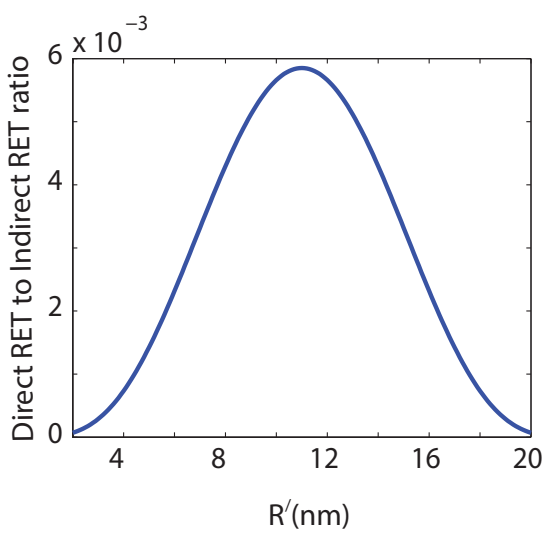

(b)

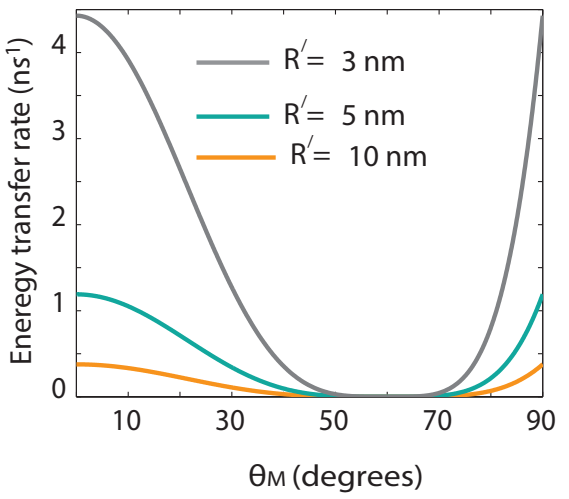

(c)

FIG. 8. Indirect RET rate: (a) as a function of the relative distance between $D$ and $M,\left(R^{\prime}\right)$, keeping $R$ constant at 20 nm; (b) as a ratio of direct RET with respect to $R^{\prime}$, keeping $R$ constant at $20 \mathrm{~nm}$; (c) as a function of $\theta_{M}$ for three different relative distances $(3 \mathrm{~nm}, 5 \mathrm{~nm}, 10 \mathrm{~nm}$ ) between $D$ and $M$.

diated only via Coulombic interactions. Therefore, the values of quantum amplitude and energy transfer rate in this region should not be regarded as physically significant (also in Dexter zone, expressions presented in our work are less meaningful as the neighbouring matter could no longer be regarded as an electronically separate entities).

\section{A. Distance dependance of indirect RET rate}

From the matrix element for indirect RET given in Eq. (23), it can be seen that the RDDI depends on relative position of all three particles, exhibiting inverse square power dependence on each of the donor and acceptor distances relative to body $M$. Moreover, compared to the direct interaction of two NWs derived in Eq. (16), creation and annihilation of two virtual photons reduce the quantum amplitude of the indirect interaction, displaying a $\left(R^{\prime}\right)^{-4}\left(R^{\prime \prime}\right)^{-4}$ distance dependence of indirect RET rate (see Eq.27). Furthermore, indirect RET rate gradually decreases as a function of displacement of $A$ from $M$ and $D$ (keeping $M$ at the midpoint of the $D-A$ axis). In addition, indirect transfer rate is less distinguishable when $M$ is located in the center of the $D$ - $A$ axis, as shown in Fig.8 (a).

We now focus on the important possibility of altering the energy transfer rate by indirect energy transfer, by analysing the ratio of the, direct to indirect transfer rates. This is depicted in Fig.8 (b) for various $R^{\prime}$ values at fixed $R$. It can be observed that the influence of the included intermediary $\mathrm{NW},(M)$, becomes more prominent when it is situated close to either donor or acceptor.

\section{B. Orientational dependance of indirect $\mathrm{RET}$ rate}

The relative orientation of the $D, M, A$, and their individual orientations with respect to relative separation vectors, strongly influence the quantum amplitude $\left(M_{F I}^{i} \propto\left|\kappa_{D M}\right|\left|\kappa_{M A}\right|\right)$ and the indirect energy transfer rate $\left(\Gamma_{\text {tran }}^{i} \propto\left|\kappa_{D M}\right|^{2}\left|\kappa_{M A}\right|^{2}\right)$. We now conduct a comprehensive analysis of how the transfer rate varies as a function of $\theta_{M}$ for various $R^{\prime}$ and $R$ values. As illustrated in Fig. 8 (c), the orientation dependence of rate becomes substantial when $M$ gets closer to either the acceptor or donor. The orientation factor is less significant when the passive NW is placed at the center of the donor-acceptor displacement vector. Moreover, analogous to the direct energy transfer, orientational factor becomes stronger when the acceptor is placed near to the donor.

The angles given in Eq. (25) and Eq. (26) are dependent on one another. Once again we identify three non-trivial cases to study the orientational dependence of third-body modified energy transfer.

1. case 1: $\theta_{D M}=\theta_{M A}=0, \theta_{D}^{\prime}=\theta_{A}^{\prime}=\theta_{M}=\theta_{M}^{\prime}=0$; $\kappa_{D M}=\kappa_{M A}=-1$, (Fig. 9 (a))

2. case 2: $\theta_{D M}=\theta_{M A}=0, \theta_{D}^{\prime}=\theta_{A}^{\prime}=0, \theta_{M}=\theta_{M}^{\prime}=$ $\frac{\pi}{3} ; \kappa_{D M}=\kappa_{M A}=0$, (Fig. 9 (b))

3. case 3: $\theta_{D M}=\theta_{M A}=\frac{\pi}{3}, \theta_{D}^{\prime}=\theta_{A}^{\prime}=\theta_{M}=\theta_{M}^{\prime}=$ $\frac{\pi}{3} ; \kappa_{D M}=\kappa_{M A}=0,($ Fig. 9 (c))

In all three cases $\theta_{D A}=\theta_{D}=\theta_{A}=0$.

The first case corresponds to situations when the transition dipole moments are parallel (or antiparallel) to each other and separation vectors, leading to $\left|\kappa_{D M}\right|^{2}=$ $\left|\kappa_{M A}\right|^{2}=1$. These geometrical configuration of three NWs gives highest indirect RET rate. In the second case, we keep all angles unaltered except $\theta_{M}, \theta_{M}^{\prime}$, here the dipole moment of $M$ formes angle $\frac{\pi}{3}$ with respect to both 


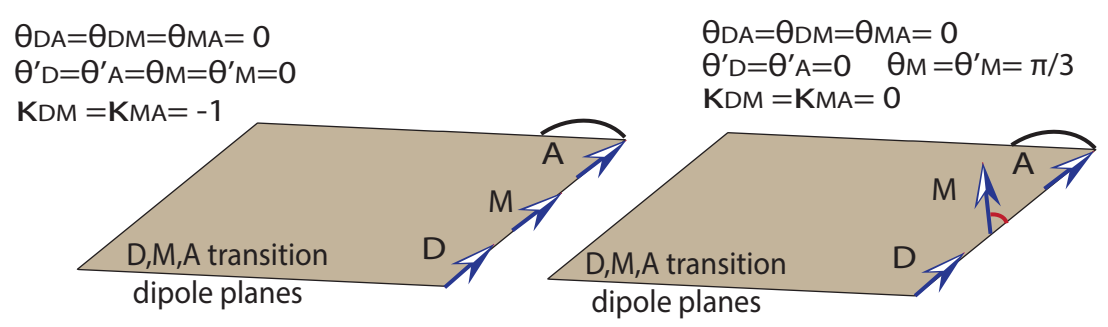

(a)

(b)

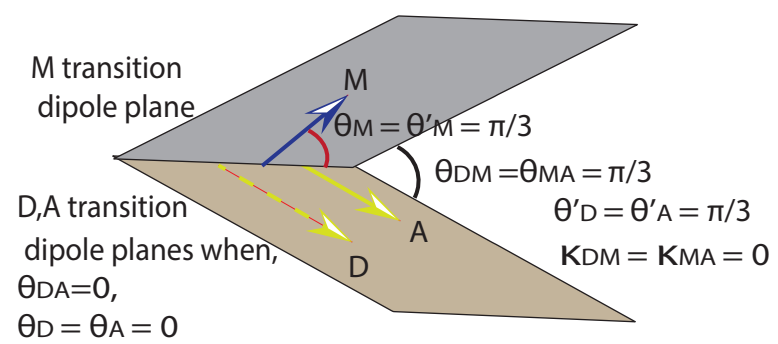

(c)

FIG. 9. Indirect RET orientation factor in Eq. (25) and Eq. (26): (a) $M$ lies in the D-A separation vector, and all $D, M, A$ are parallel to each other; (b) $M$ lies in the D-A separation vector, $\theta_{M}=\theta_{M}^{\prime}=\frac{\pi}{3}$, and $\theta_{D M}=\theta_{M A}=0$; (c) $\theta_{D A}=\frac{\pi}{3}$, $\theta_{M}=\theta_{M}^{\prime}=\theta_{D}^{\prime}=\theta_{A}^{\prime}=\frac{\pi}{3}$. Note that in all cases $\theta_{D A}=\theta_{D}=\theta_{A}=0$

$\boldsymbol{R}^{\prime}, \boldsymbol{R}^{\prime \prime}$, leading to a prohibited indirect energy transfer rate. The case three is similar to the case two, except the plane of $M$ makes an angle of $\frac{\pi}{3}$ with respect to donor and acceptor transition dipole planes. This configuration of the NW system makes indirect RET rate contribution to the total energy transfer rate to be vanished.

\section{Quantum interference contribution to the transfer rate}

From the matrix elements for direct, and indirect transfer, it is straightforward to calculate the third term of Eq. (8), that arises from the interference of these two processes.

$$
\begin{aligned}
\Gamma_{\text {tran }}^{i n t} & =\frac{4 \pi \rho}{\hbar} \operatorname{Re}\left\{\bar{M}_{F I}^{d} M_{F I}^{i}\right\} \\
& =-\frac{\left|\mu^{0 \alpha}(D)\right|^{2}\left|\mu^{\beta 0}(A)\right|^{2} \kappa_{D A} \kappa_{D M} \kappa_{M A}\left|\alpha_{k l}(M ; k)\right| \rho}{2 \pi^{2} L^{3} R^{2} R^{\prime 2} R^{\prime \prime 2} \epsilon_{0}{ }^{3} \hbar}
\end{aligned}
$$

The quantum interference, Eq. (28), displays the inverse square power dependence on each relative displacement component $\left(R, R^{\prime}, R^{\prime \prime}\right)$. Fig. 10 (a) shows the interference dependence as a function of the position of the third NW. As can be anticipated, it is clear from the plot that the quantum interference acquires higher values for lower or higher values of $R^{\prime}$, delivering minimum direct to interference ratio when $M$ is at the center of the $D-A$ displacement, as illustrated in Fig. 10 (b).

Inspection of Fig. 10 (c) allows one to gain insight into how the interference term is influenced by the rela- tive orientation of particles, exhibiting the quantum interference variation with respect to $\theta_{M}$ when $\theta_{D M}=0, \pi$. In both cases, quantum interference is maximum when $\theta_{M}=\frac{\pi}{3}$, and minimum when $\theta_{M}=0, \frac{\pi}{2}$. For a collinear arrangement of the three NWs, the quantum interference between direct and indirect transfer can be negative, decreasing the total exchange rate.

Thus, the total third-body-modified energy transfer rate becomes

$$
\begin{array}{r}
\Gamma_{\text {tran }}^{\text {total }}=\frac{\left|\mu^{0 \alpha}(D)\right|^{2}\left|\mu^{\beta 0}(A)\right|^{2}|\kappa|^{2} \rho}{2 \pi L^{2} R^{4} \epsilon_{0}{ }^{2} \hbar}+ \\
\frac{\left|\mu^{0 \alpha}(D)\right|^{2}\left|\mu^{\beta 0}(A)\right|^{2}\left|\kappa_{D M}\right|^{2}\left|\kappa_{M A}\right|^{2}\left|\alpha_{k l}(M ; k)\right|^{2} \rho}{8 \pi \hbar L^{4} R^{\prime 4} R^{\prime \prime 4} \epsilon_{0}{ }^{4}}- \\
\frac{\left|\mu^{0 \alpha}(D)\right|^{2}\left|\mu^{\beta 0}(A)\right|^{2} \kappa_{D A} \kappa_{D M} \kappa_{M A}\left|\alpha_{k l}(M ; k)\right| \rho}{2 \pi^{2} L^{3} R^{2} R^{\prime 2} R^{\prime \prime 2} \epsilon_{0}{ }^{3} \hbar}
\end{array}
$$

The result, Eq. (29) can be interpreted as the rate for RET between donor and acceptor NWs, modified by the presence of the passively interacting medium.

\section{DISCUSSION}

We have derived resonance energy transfer rate equations for a system consisting of nanowires of the same dimensionality. The calculations are presented for direct RET and the influence of a passive NW on the energy transfer rate. The results have demonstrated the relative spacing between NWs along with the relative orientation of the transition dipoles determine the controllability of 


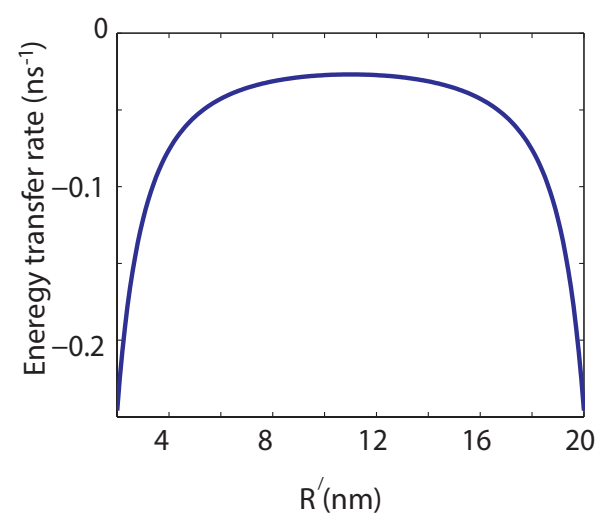

(a)

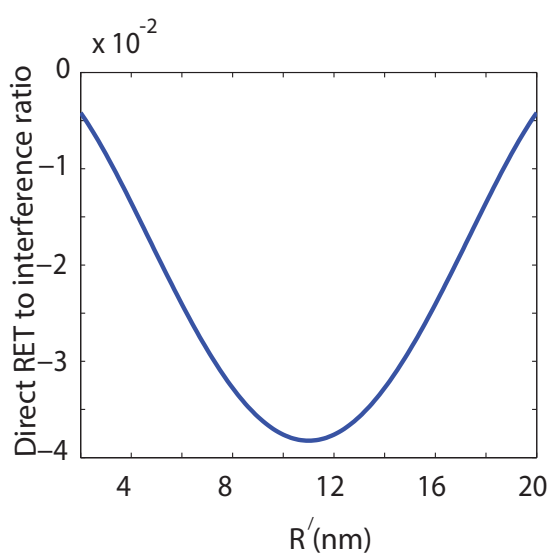

(b)

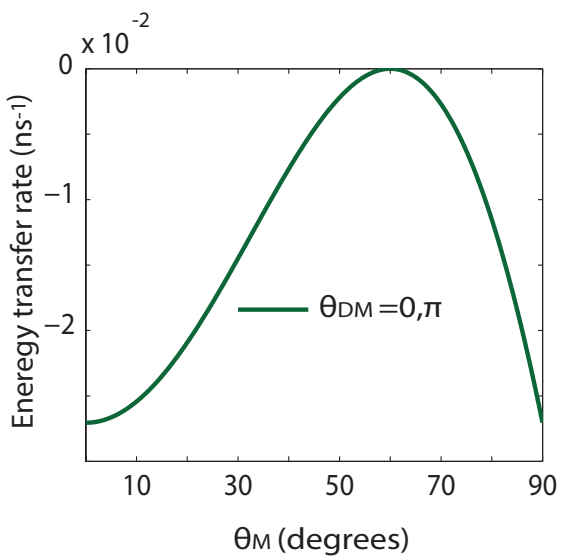

(c)

FIG. 10. Quantum interference: (a) as a function of the relative distance between D and M $\left(R^{\prime}\right)$, keeping $R$ constant at $20 \mathrm{~nm}$; (b) as a ratio of direct RET with respect to $R^{\prime}$, keeping $R$ constant at $20 \mathrm{~nm}$; (c) variation as a function of $\theta_{M}$ when $\theta_{D M}=0$ and $\theta_{D M}=\pi$

resonance energy transfer rate. Furthermore, the RET in NW systems displays a slower spatial decay compares to a system consisted of QDs.

In Sec. III, unmediated RET between two NWs has been studied, applying the second order perturbation theory. The results have demonstrated the energy transfer rate exhibits $R^{-4}$ distance dependence. The orientation factor, $|\kappa|^{2}$ varies from 0 to 1 . Moreover, in Sec. IV third body-mediated RET has been investigated, exploiting the fourth order perturbation mechanism. Here, the passive NW, $M$, remains in its ground state but is excited in intermediate states. It participates in the transfer of excitation as a polarizable body coupled to the electromagnetic field. Therefore, the energy transfer rate embraces additional contributions associated with indirect RET rate and quantum interference. We obtained analytical expressions for both indirect energy transfer rate and quantum interference contribution to the total third-body mediated RET rate. The indirect coupling of resonance energy transfer becomes more significant as the density of particles $M$ increases. In a nutshell, these results suggest that when designing an artificial energy transfer system, optimal configurations for fast transfer between nearby sites are those where the NW transition dipole moments and the separation vector are collinear. However, coupling may also be "switched off" between particles by arranging them such that the transition dipole moments are perpendicular to each other. This can, in effect, enable one to design a nano-antenna system that are optimized to focus the energy transfer to specific points. Moreover, an interacting third NW can effectively enhance or inhibit the RET between donor and acceptor, and this contribution can be significant if a sufficient number of passive objects $(M)$ are present.

\section{CONCLUSIONS}

In this article, the direct RET between two NWs and the influence of a passively interacting NW have been studied using the theory of molecular quantum electrodynamics. Within the QED context, treatment of resonance energy transfer in both cases, exchange of excitation is mediated by electromagnetic signals propagating at the speed of light. Thus, the coupling matrix elements and rate equations are derived for both cases, and the intricate interplay of the relative distance, orientation, and the effect of the passively interacting medium on the transfer efficiency have been studied to a greater extent.

Summarizing, the ensuing results demonstrated the possibility of altering the strength and the directivity of the resonance energy transfer between two NWs by careful engineering of the spacing, orientation and inclusion of an additional quantum objects in the vicinity. The analysis thus provides pointers to the means of controlling and optimizing the transfer of energy between discrete components, potentially in any multi-nanowire system, inviting surface and layer applications. Therefore, this research opens up substantial opportunities to develop a thorough understanding on well-known RET mechanism in NWs, based on quantum electrodynamics. In particular, the results we have secured should support the further improvement of design of biological sensors, organic photovoltaics and light-driven catalysis, optical switching, through the achievement of new methods for optically controlled transmission.

\section{ACKNOWLEDGMENTS}

The work of D.W. is supported by the Monash University Institute of Graduate Research. The work of M.P. is 
supported by the Australian Research Council, through its Discovery Grant No. DP140100883.
[1] A. A. Lamola, Photochemistry and photobiology 8, 601 (1968).

[2] H. Park, N. Heldman, P. Rebentrost, L. Abbondanza, A. Iagatti, A. Alessi, B. Patrizi, M. Salvalaggio, L. Bussotti, M. Mohseni, et al., Nature materials (2015).

[3] J. Strumpfer, M. Sener, and K. Schulten, J. Phys. Chem. Lett. 3, 536 (2012).

[4] D. Sikdar, W. Cheng, and M. Premaratne, J. App. Phy. 117, 083101 (2015).

[5] C. Rupasinghe, I. D. Rukhlenko, and M. Premaratne, ACS nano 8, 2431 (2014).

[6] C. Jayasekara, M. Premaratne, M. I. Stockman, and S. D. Gunapala, J. App. Phy. 118, 173101 (2015).

[7] S. Chatterjee, J. B. Lee, N. V. Valappil, D. Luo, and V. M. Menon, Bio. Opt. Express 2, 1727 (2011).

[8] A. I. Hochbaum and P. Yang (ACS Publications, 2009) pp. 527-546.

[9] K. Shankar, X. Feng, and C. A. Grimes, Acs Nano 3, 788 (2009).

[10] S. Lu, Z. Lingley, T. Asano, D. Harris, T. Barwicz, S. Guha, and A. Madhukar, Nano letters 9, 4548 (2009).

[11] K. Becker, J. M. Lupton, J. Müller, A. L. Rogach, D. V. Talapin, H. Weller, and J. Feldmann, Nature Materials 5, 777 (2006).

[12] D. L. Andrews, Phy. Rev. Lett. 99, 023601 (2007).

[13] M. Premaratne and G. P. Agrawal, Light Propagation in Gain Media (Cambridge University Press, 2011).

[14] X. Liu and J. Qiu, Chemical Society Reviews 44, 8714 (2015).

[15] K. Tomioka, M. Yoshimura, and T. Fukui, Nature 488, 189 (2012).

[16] T. Larsen, K. Petersson, F. Kuemmeth, T. Jespersen, P. Krogstrup, J. Nygard, and C. Marcus, Phys. Rev. Lett. (2015).

[17] Y. Xia, P. Yang, Y. Sun, Y. Wu, B. Mayers, B. Gates, Y. Yin, F. Kim, and H. Yan, Adv. materials 15, 353 (2003).

[18] X. Duan, Y. Huang, Y. Cui, J. Wang, and C. M. Lieber, Nature 409, 66 (2001).

[19] Y. P. Rakovich, F. Jäckel, J. F. Donegan, and A. L. Rogach, J. Mat. Chem. 22, 20831 (2012).

[20] I. D. Rukhlenko, D. Handapangoda, M. Premaratne, A. V. Fedorov, A. V. Baranov, and C. Jagadish, Optics express 17, 17570 (2009).

[21] M. Artemyev, E. Ustinovich, and I. Nabiev, J. Ame. Chem. Soc. 131, 8061 (2009).

[22] P. L. Hernández-Martínez and A. O. Govorov, Phys. Rev. B 78, 035314 (2008).

[23] C. S. Kumarasinghe, M. Premaratne, Q. Bao, and G. P. Agrawal, Scientific reports 5 (2015).
[24] P. L. Hernández-Martínez and A. O. Govorov, Phys. Rev. B 78, 035314 (2008).

[25] R. El-Ganainy and S. John, New J. Phys 15, 083033 (2013).

[26] R. Swathi and K. Sebastian, J. Chem. Phys. 132, 104502 (2010).

[27] D. L. Andrews and D. S. Bradshaw, Eur. J. Phys. 25, 845 (2004).

[28] D. P. Craig and T. Thirunamachandran, Molecular Quantum Electrodynamics (Dover, New York, 1998).

[29] D. L. Andrews and A. A. Demidov, Resonance energy transfer (Wiley, 1999).

[30] D. L. Andrews, C. Curutchet, and G. D. Scholes, Laser and Photonics Reviews 5, 114 (2011).

[31] E. F. James and A. J. Garth, New Journal of Physics 16, 113067 (2014).

[32] A. Salam, J. Chem. Phys. 122, 044112 (2005).

[33] A. Salam, Molecular quantum electrodynamics: longrange intermolecular interactions (John Wiley \& Sons, 2010).

[34] G. Juzeliūnas and D. L. Andrews, Phys. Rev. B 50, 13371 (1994).

[35] D. L. Andrews, Chem. Phy. 135, 195 (1989).

[36] G. J. Daniels, R. D. Jenkins, D. S. Bradshaw, and D. L. Andrews, J. Chem. Phys. 119, 2264 (2003).

[37] D. Weeraddana, M. Premaratne, and D. L. Andrews, Phys. Rev. B 92, 035128 (2015).

[38] G. D. Scholes and D. L. Andrews, The Journal of Chemical Physics 107 (1997).

[39] R. P. Feynman, Phys. Rev. 94, 262 (1954).

[40] A. Salam, J. Chem. Phys. 136, 014509 (2012).

[41] D. L. Andrews and J. S. Ford, J. Chem. Phys. 139, 014107 (2013).

[42] D. P. Craig and T. Thirunamachandran, Chem. Phys. 135, 37 (1989).

[43] P. Berman, R. W. Boyd, and P. W. Milonni, Phys. Rev. A 74, 053816 (2006).

[44] P. Milonni, R. Loudon, P. R. Berman, and S. M. Barnett, Phys. Rev. A 77, 043835 (2008).

[45] F. Frezza, L. Pajewski, D. Saccoccioni, and G. Schettini, Optics Communications 265, 47 (2006).

[46] R. Bennett, T. M. Barlow, and A. Beige, Eur. J. Phys. 37, 014001 (2015).

[47] M. Abramowitz and I. A. Stegun, Handbook of mathematical functions: with formulas, graphs, and mathematical tables, 55 (Courier Corporation, 1964).

[48] G. J. Daniels and D. L. Andrews, The Journal of Chemical Physics 117, 6882 (2002).

[49] T. Förster, Annalen der Physik 437, 55 (1948).

[50] G. D. Scholes and D. L. Andrews, Phys. Rev. B 72, $125331(2005)$ 